\title{
OPTIMAL DIAMETER ASSIGNMENT FOR GAS PIPELINE NETWORKS
}

\author{
SITA BHASKARAN and FRANZ J. M. SALZBORN
}

(Received 19 June 1978)

(Revised 19 October 1978)

\begin{abstract}
A linear programming model for optimally assigning diameters to a gas pipeline network is discussed. Computational results for a real life situation are presented, and certain properties that have to be satisfied by an optimal assignment are derived.
\end{abstract}

\section{Introduction}

This paper is concerned with a gas pipeline network that transports gas from a set of wells to a factory prior to dispatch along a main trunk line. In designing such a network several stages can be distinguished. Firstly, one has to determine the configuration of the network; in other words, one has to decide how the wells and the plant will be connected up and which junction nodes, where pipes are jointed together, have to be inserted, as junctions do not necessarily occur at the wells. This is called the configuration problem. Then the exact position of the junction nodes has to be established: the junction location problem. Finally, the diameter of each of the links has to be determined: the diameter assignment problem. This last problem is the subject of this paper. In another forthcoming paper [2] by the same authors the first two problems are addressed, however in a slightly less general context than that adopted here.

The problem considered here is thus: given a number of wells, for each of which a forecast of the output gas flow is available over a number of years, and given the configuration and position of the pipeline network, how does one select the diameters of each of the links such that the total cost of the network is minimized.

(C) Copyright Australian Mathematical Society 1979

Copyright. Apart from any fair dealing for scholarly purposes as permitted under the Copyright Act, no part of this JOURNAL may be reproduced by any process without written permission from the Treasurer of the Australian Mathematical Society. 
The pipeline diameters will determine the pressure drops between the plant and each of the wells. The constraints on the diameters are caused by the restriction that these pressure drops are not allowed to exceed a certain limit: the gas has to arrive at the plant at a certain pressure and there is a maximum for the pressure at which the gas can be delivered at the wells. The diameters are selected from a finite set of possible diameters, for each of which the cost per mile is given. But it is possible for a link to consist of sections of different diameters connected in series.

It will be assumed throughout this paper that the network under consideration is a tree; in other words, that it contains no meshes. The justification for this restriction can be found in [2], where it is proved that under certain reasonable conditions the optimal configuration is a tree network.

This study was initiated by an investigation of the Moomba gasfield in the desert centre of Australia; the Delhi Corporation provided us with the necessary data.

The paper presents a linear programming model for the diameter assignment problem which results in an efficient algorithm for finding the optimal diameters; computer time for handling a real world situation is a few seconds. In addition some properties of optimal diameter assignments are derived.

(i) At most two pipe sizes are used for one link.

(ii) Under fairly general conditions the following result holds: if for any link two pipe sizes are used, then they must be of consecutive sizes.

(iii) If the specific gravity of gas is the same for all wells then there exists an optimal diameter assignment in which diameters do not decrease in the direction of flow.

Papers that deal with pipeline construction are Rothfarb et al. [5] and Zadeh [7]. They, however, consider an offshore pipeline system, for which junctions other than at existing well rigs are not possible and only one time period. Both Rothfarb et al. and Zadeh use for the optimal assignment of diameters a dynamic programming approach and Zadeh has also used a minimum cost flow approach.

\section{Basic concepts and definitions}

\section{Flow formula}

In the design of gas pipeline networks the formula used to describe the flow of gas through pipelines is of vital importance. There exist empirical formulae giving the pressure square drop per unit length of pipe as a function of flow, specific gravity and diameter:

$$
\frac{p p}{l}=f(q, s, d)
$$


where

$q=$ rate of gas flow,

$s=$ specific gravity of flowing gas,

$l=$ length of the pipe,

$d=$ internal diameter of the pipe,

$p p=$ pressure square drop between the ends of the pipe.

All variables $q, s, d, p p$ and $l$ are nonnegative.

We are not going into the experimental details of deriving the formula $f$. We assume that $f$ is a given monomial:

$$
f(q, s, d)=M \frac{q^{\alpha_{1}} s^{\alpha_{2}}}{d^{\alpha_{2}}},
$$

where $M, \alpha_{1}, \alpha_{2} \alpha_{3}$ are positive constants. The value of $M$ depends upon the units of measurement used.

Of the existing empirical formulae, we have used, for our numerical computations, the Weymouth formula which is popular for the range of pressures we have to deal with.

$$
\text { Weymouth formula: } \frac{p p}{l}=f(q, s, d)=M \frac{q^{2} s}{d^{16 / 3}} .
$$

The value of $M$, for the units we use, is given with the computational results.

To maintain generality, we will, where possible, use the general form (2) of the monomial $f$ instead of the specific formula (3).

\section{Tree networks}

The elements of a tree network are:

A: The nodes of the tree, which can be classified as follows:

(1) the set $N_{f}=\{0,1, \ldots, m\}$ which includes the plant (0) and the wells;

(2) the set $N_{s}$ of extra junction nodes where different pipe sections are joined together.

We define $N=N_{f} \cup N$ and $\bar{N}=N \backslash\{0\}$.

The positions $(x(i), y(i)), i \in N$, are given.

B : The links of the tree which can be specified by defining a predecessor function

$$
a: \bar{N} \rightarrow N \text {. }
$$

The set of links is thus

$$
A_{a}=\{(a(i), i) \mid i \in \bar{N}\} .
$$

The function $a$ must be such that the graph $\left[N, A_{a}\right]$ contains no meshes.

The set of successor nodes of node $i$ is defined by

$$
B(i)=\{j \in N \mid a(j)=i\} \quad \text { for all } i \in N,
$$


and

$$
\theta=\{j \in N \mid B(j)=\varnothing\}
$$

denotes the set of extremal nodes of the tree. Note that $\theta \subset \bar{N}_{f}:$ all extremal nodes are wells.

The diameters of each link of the network are selected from a finite set:

$$
D=\left\{d_{1}, d_{2}, \ldots, d_{n}\right\}
$$

For each $d \in D$ a cost per unit length $C(d)$ is specified. $C(d)$ is always a monotonically increasing function of $d$.

Finally, we define

$$
\left.l(i)=\left[(x(i)-x(a(i)))^{2}+(y(i)-y(a(i)))^{2}\right)\right]^{1},
$$

the length of link $i$. The values of $l(i)$ can be calculated directly from the given data.

\section{Flows and pressures}

The following values are given:

$P_{0}$-a fixed pressure at which the gas must be delivered at the plant, and $P_{1}$-the maximum pressure at which gas can be provided at the wells.

Let a period of $T$ years be considered. For year $t, t=1,2, \ldots, T$, we define:

$Q(i, t)$-rate of flow of gas produced at well $i$ in year $t$, where $i \in N_{f} \backslash\{0\}$, $S(i, t)$-specific gravity of gas produced at well $i$ in year $t$, where $i \in N_{f} \backslash\{0\}$, $q(i, t)$-rate of flow of gas in link $(a(i), i)$ in year $t$, where $i \in \bar{N}$, $s(i, t)$-specific gravity of gas in link $(a(i), i)$ in year $t$, where $i \in \bar{N}$, $p(i, t)$-pressure at node $i$ in year $t$, where $i \in N$, $p p(i, t)$-pressure square drop on link $(a(i), i)$ in year $t$, where $i \in \bar{N}$. Thus, $p p(i, t)=p^{2}(i, t)-p^{2}(a(i), t)$.

For each link $(a(i), i) \in A_{a}$, for every year $t=1,2, \ldots, T$, the flow rates are given by

$$
\begin{aligned}
& q(i, t)=Q(i, t), \quad \text { for extreme nodes } i \in \theta, \\
& q(i, t)=\sum_{j \in B(i)} q(j, t)+Q(i, t), \quad \text { if } i \notin \theta
\end{aligned}
$$

and the specific gravities are

$$
\begin{aligned}
& s(i, t)=S(i, t), \quad \text { if } i \in \theta, \\
& s(i, t)=\left[\sum_{j \in B(i)}(s(j, t) q(j, t))+S(i, t) Q(i, t)\right] /\left[\sum_{j \in B(i)} q(j, t)+Q(i, t)\right], \quad \text { if } i \notin \theta .
\end{aligned}
$$

The pressure constraints the network has to satisfy are the upper bound on pressure square drop. By (1), the pressure square drop on link $(a(i), i)$, in year $t$, 
is given by

$$
p p(i, t)=l(i) f(q(i, t), s(i, t), d(i)) .
$$

As lengths, flows and specific gravities are given quantities, the diameter of a link determines the pressure square drop. The diameters have to be chosen such that for every extreme node $i \in \theta$,

$$
\sum_{j=1}^{l} p p\left(i_{j}, t\right) \leqslant P_{1}^{2}-P_{0}^{2}, \quad \text { for } t=1, \ldots, T,
$$

where $0=i_{0}, i_{1}, \ldots, i_{k}=i$ is the path linking $i$ with 0 . Because the network is a tree with the plant $i=0$ at its origin, each of the other nodes can be reached from 0 by exactly one path.

\section{Linear programming model}

Instead of requiring the entire length of a link to be of the same diameter, we can assign to a link sections of different pipe sizes connected in series. The model we now present has the facility to do this.

The decision variables are defined as

$\delta(d, i)$ : proportion of length of link $(a(i), i)$ that has diameter $d \in D$.

These variables must satisfy

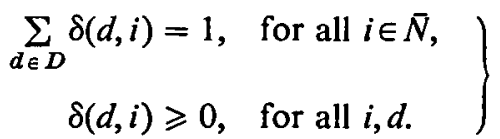

For every extreme node $i \in \theta$, let

$$
0=i_{0}, i_{1}, \ldots, i_{k}=i
$$

be the path linking $i$ with 0 . Using (1) and (2) we can express the pressure square drop on link $\left(i_{j-1}, i_{j}\right)$ in terms of the variables $\delta\left(d, i_{j}\right)$ as follows:

$$
p p\left(i_{j}, t\right)=r\left(i_{j}, t\right) \sum_{d \in D}\left(\delta\left(d, i_{j}\right) / d^{\alpha_{3}}\right)
$$

where

$$
r\left(i_{j}, t\right)=M q^{\alpha_{1}}\left(i_{j}, t\right) s^{\alpha_{2}}\left(i_{j}, t\right) l\left(i_{j}\right) .
$$

As the quantities $r\left(i_{j}, t\right)$ are known, the pressure constraints can be expressed as linear constraints in the $\delta$ 's:

$$
\sum_{j=1}^{k} \sum_{d \in D} \delta\left(d, i_{j}\right)\left(r\left(i_{j}, t\right) / d^{\alpha_{3}}\right) \leqslant P_{1}^{2}-P_{0}^{2} .
$$

We get one such constraint for each $i \in \theta$ and for each $t \in\{1, \ldots, T\}$. 
The total cost of the network can also be expressed linearly in the $\delta$ 's:

$$
\operatorname{cost}=\sum_{i \in \bar{N}} \sum_{d \in D} C(d) l(i) \delta(d, i) .
$$

An optimal diameter assignment is therefore found by solving the linear program: Minimize (7) under the constraints (5) and (6). We will call this linear program LP. The number of variables in this program is the number of links multiplicd by the number of possible diameters. The number of constraints is the number of extreme nodes multiplied by the number of years considered.

If each link is required to have one diameter over its whole length then we must add the constraint:

$$
\delta(d, i)=0 \text { or } 1
$$

and the problem turns into a $0-1$ program. We call this integer program IP.

\section{Moomba gas field: computations}

The models have been tested on data from the Moomba gas field in the South Cooper Basin in the desert of Australia. This gas field, consisting of 13 gas wells, is being developed and is spread over an area of about 4000 square miles. A map of the gas wells is given in Fig. 1. A network of pipes has to be constructed to

$$
\begin{aligned}
& \text { 11-Fly Lake } \\
& 10 \text {-Tirrawarra } \\
& \text { 9-Merrimelia } \\
& \begin{array}{l}
\text { 0-MOOMBA 2-Della } \\
\text { PLANT }
\end{array} \\
& \text { 1-Big Lake } \\
& \begin{array}{l}
\text {-Dullingari } \\
\text { 5-Burke } \quad \text { 8-Esilon } \\
\text { 8-Roseneath }
\end{array} \\
& \text { 13-Daralingie } \\
& \begin{array}{l}
\text { 3-Toolachee } \\
\text { 6-Brumby }
\end{array}
\end{aligned}
$$

Fig. 1. Moomba gas field: location of the wells. 


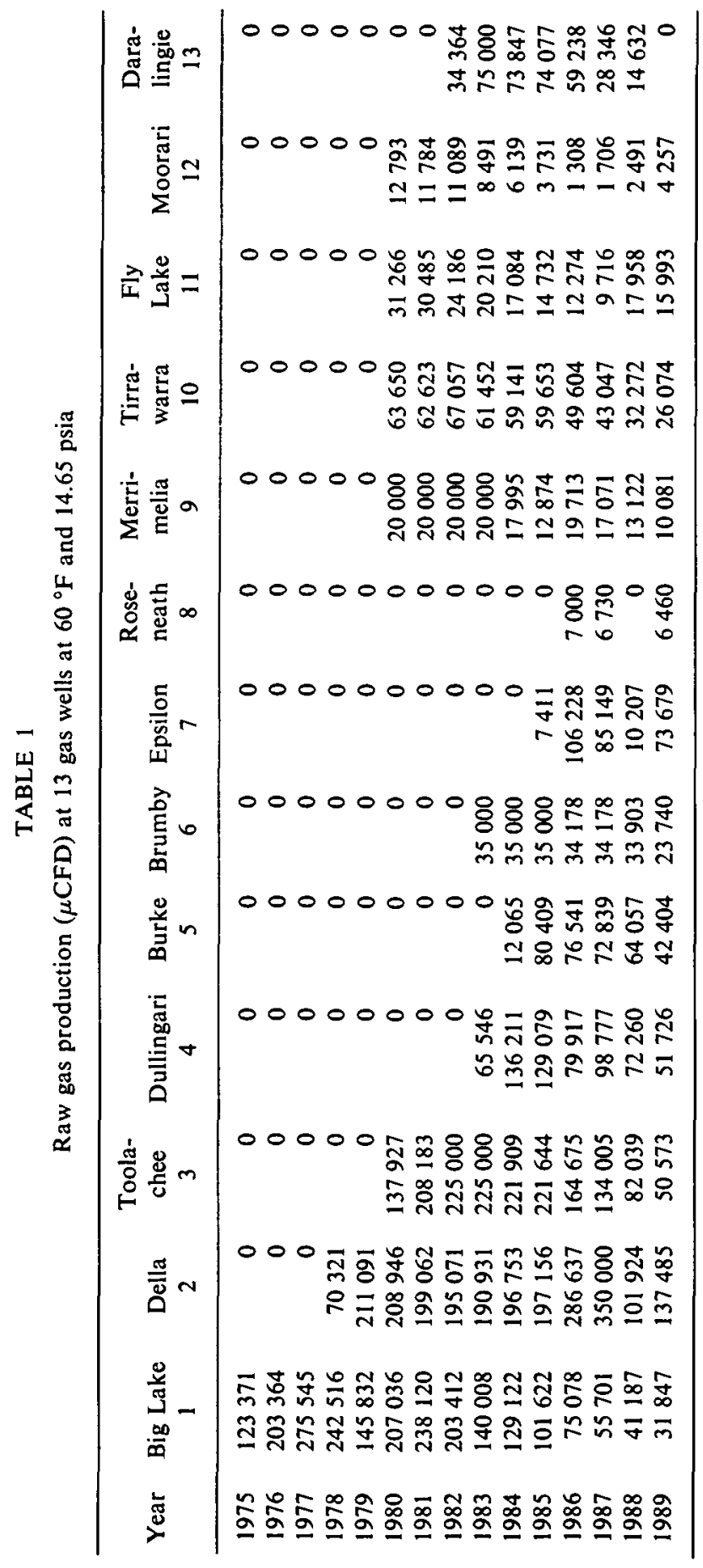




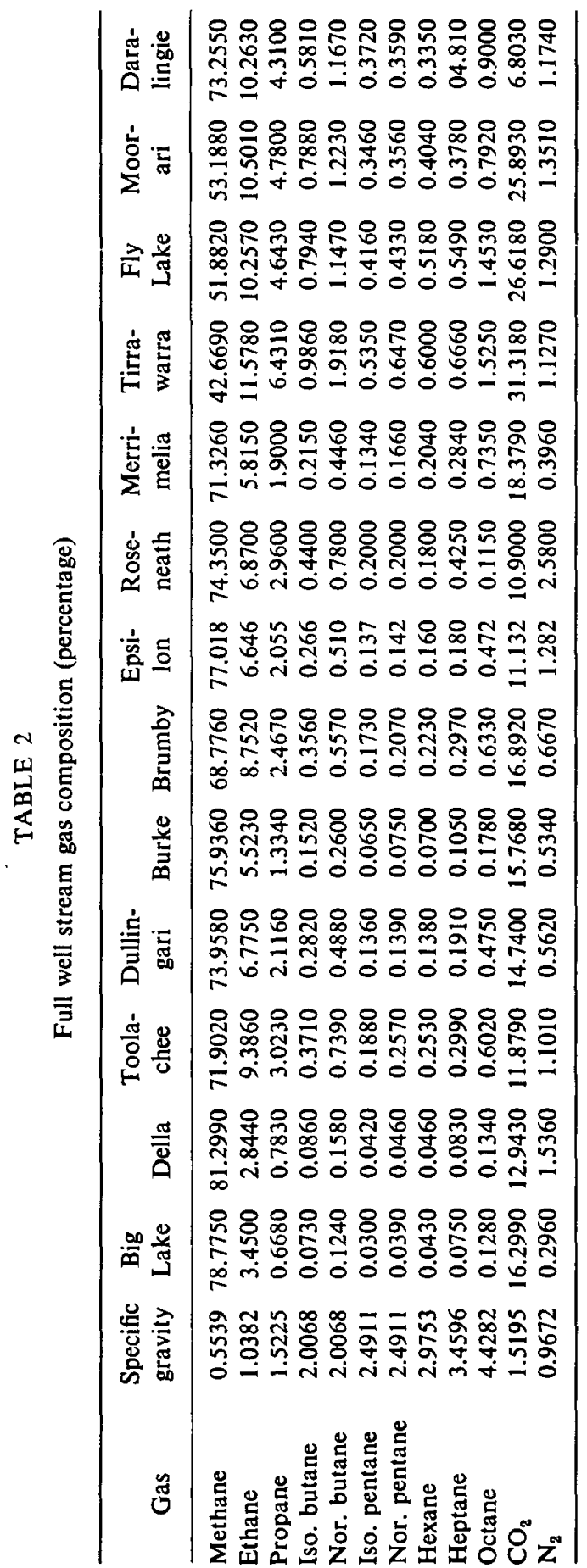


transport gas from the wells to the factory prior to entry into a trunk main for onward transmission. The critical gas wells which are now under active consideration are the wells numbered 0 to 8 . We restrict our computational results to this section of the gas field.

The data relating to the Moomba field are given in Tables 1, 2 and 3. The units used are: flow in cu. ft per 24 hours, pressure in lb per sq. inch absolute, length of links in miles, diameter of pipes in inches. An estimated rate of raw gas production during the years $1975-89$ is given in Table 1 . The full well stream

TABLE 3

Pipeline dimensions and costs

\begin{tabular}{ccc}
\hline Pipe size & $\begin{array}{c}\text { Internal diameter } \\
\text { (inches) }\end{array}$ & $\begin{array}{c}\text { Pipe cost } \\
(\$ / \text { mile })\end{array}$ \\
\hline 1 & 4.000 & 28200 \\
2 & 6.125 & 40500 \\
3 & 8.001 & 50080 \\
4 & 10.136 & 59,200 \\
5 & 12.062 & 73680 \\
6 & 13.250 & 100800 \\
7 & 15.250 & 135680 \\
8 & 17.250 & 176220 \\
9 & 19.188 & 222000 \\
10 & 21.124 & 250800 \\
11 & 23.062 & 281760 \\
12 & 25.062 & 314600 \\
13 & 27.000 & 339000 \\
14 & 28.876 & 364000 \\
15 & 30.876 & 388000 \\
16 & 32.876 & 411000 \\
17 & 34.750 & 431000 \\
18 & 36.750 & 451000 \\
19 & 38.750 & 470000 \\
\hline
\end{tabular}

gas compositions for the various wells and the specific gravities of the components are given in Table 2 . The specific gravity of emerging gas is the weighted specific gravity of the components.

The pressure limits $P_{0}$ and $P_{1}$ are given to be 1115 and $1185 \mathrm{lb} / \mathrm{sq}$. inch respectively. There are 19 pipe sizes, each size being characterized by its internal diameter and the associated cost per mile. This is given in Table 3.

The Weymouth formula is

$$
\frac{p p}{l}=f(q, s, d)=M \frac{q^{2} s}{d^{16 / 3}} .
$$


The constant $M$ is given by

$$
M=\frac{1}{(433.45)}\left(\frac{P_{s}}{T_{s}}\right)^{2} T
$$

where

$T$ is absolute temperature of flowing gas $\left({ }^{\circ} \mathrm{F}+460\right)$,

$T_{g}$ is standard absolute temperature,

$P_{s}$ is standard pressure (lb per sq. inch absolute).

These constants are in our case $T_{s}=520, T=560$ and $P_{s}=14.65$. The computer we use is a C.D.C. Cyber 173.

\section{Results for linear and integer programming models}

To solve the linear program LP and the integer program IP, we used APEX III, a large Control Data Corporation Linear Programming package at the University of Adelaide. APEX III has mixed integer facilities and uses a branch-and-bound technique for integer programming. We solved both LP and IP, for a wide variety of trees, both for $T=1$ (the year 1986) and also for $T=10$ (the period 1980-89).

We would like to mention here that for all optimal diameter assignments we obtained by solving LP, diameters never decreased in the direction of flow.

\section{Example 1}

The tree is given in Fig. 2. Table 4 gives the cost of this tree and the time taken by the programs when $T=1$ and when $T=10$. The lengths of the links and the various diameters assigned by the various programs are given in Table 5 .
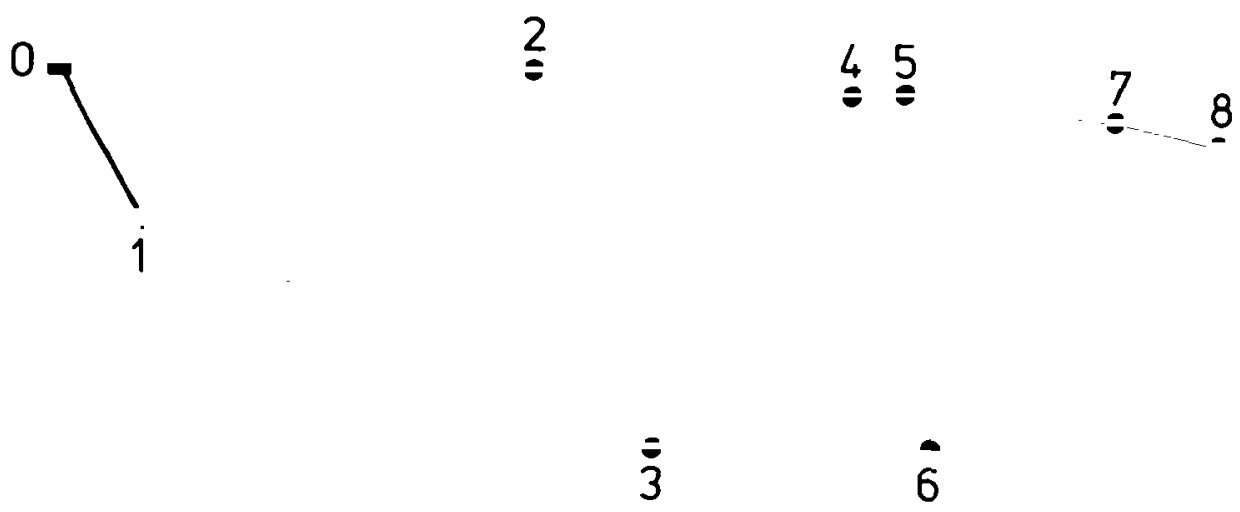

Fig. 2. Tree considered for diameter assignment in example 1. 
TABLE 4

Minimum cost of network and time taken to compute optimal assignment of example 1

\begin{tabular}{llcc}
\hline $\begin{array}{l}\text { Number } \\
\text { of years }\end{array}$ & Program & $\begin{array}{c}\text { Cost of network } \\
(\$)\end{array}$ & $\begin{array}{c}\text { Time taken } \\
\text { (CP sec) }\end{array}$ \\
\hline$T=1$ & LP & 36118307 & 2.1 \\
& IP & 36429252 & 16.3 \\
$T=10$ & LP & 37793435 & 4.1 \\
& IP & 38041214 & 23.1 \\
\hline
\end{tabular}

TABLE 5

Pipe sizes assigned to links of tree in example 1

\begin{tabular}{|c|c|c|c|c|c|}
\hline \multirow[b]{3}{*}{ Link } & \multirow{3}{*}{$\begin{array}{l}\text { Length } \\
\text { of link } \\
\text { (miles) }\end{array}$} & \multicolumn{4}{|c|}{ Pipe sizes assigned to link or proportion of link } \\
\hline & & \multicolumn{2}{|c|}{$T=1$} & \multicolumn{2}{|c|}{$T=10$} \\
\hline & & LP & IP & LP & IP \\
\hline$(0,1)$ & 9.71 & 13 & 14 & $\begin{array}{l}15(66 \%) \\
16(34 \%)\end{array}$ & 16 \\
\hline$(0,2)$ & 25.89 & $\begin{array}{l}17(59 \%) \\
18(41 \%)\end{array}$ & 18 & $\begin{array}{l}17(18 \%) \\
18(82 \%)\end{array}$ & 18 \\
\hline$(1,3)$ & 33.18 & $\begin{array}{l}11(27 \%) \\
12(73 \%)\end{array}$ & 12 & 13 & 13 \\
\hline$(2,4)$ & 17.43 & 13 & 13 & 13 & 13 \\
\hline$(3,6)$ & 12.21 & 6 & 5 & 6 & 6 \\
\hline$(4,5)$ & 2.84 & 12 & 14 & 11 & 10 \\
\hline$(5,7)$ & 11.67 & 10 & 9 & $\begin{array}{r}9(29 \%) \\
10(71 \%)\end{array}$ & 10 \\
\hline$(7,8)$ & 5.83 & 4 & 4 & 4 & 4 \\
\hline
\end{tabular}

TABLE 6

Minimum cost of network and time taken to compute optimal assignment of example 2

\begin{tabular}{llcc}
\hline $\begin{array}{l}\text { Number } \\
\text { of years }\end{array}$ & Program & $\begin{array}{c}\text { Cost of network } \\
(\$)\end{array}$ & $\begin{array}{c}\text { Time taken } \\
\text { (CP sec) }\end{array}$ \\
\hline$T=1$ & LP & 32964110 & 3.87 \\
& IP & 33515679 & 17.73 \\
$T=10$ & LP & 34559858 & 10.4 \\
& IP & 35131750 & 54.33 \\
\hline
\end{tabular}




\section{Example 2}

Tables 6 and 7 give the corresponding results for the tree in Fig. 3.

For using APEX III the data have to be in MPS format as required by the

TABLE 7

Pipe sizes assigned to links of tree in example 2

\begin{tabular}{|c|c|c|c|c|c|}
\hline \multirow[b]{3}{*}{ Link } & \multirow{3}{*}{$\begin{array}{l}\text { Length } \\
\text { of link } \\
\text { (miles) }\end{array}$} & \multicolumn{4}{|c|}{ Pipe sizes assigned to link or proportion of link } \\
\hline & & \multicolumn{2}{|c|}{$T=1$} & \multicolumn{2}{|c|}{$T=10$} \\
\hline & & $\mathbf{L P}$ & IP & LP & IP \\
\hline$(0,9)$ & 4.46 & 19 & 19 & 19 & 19 \\
\hline$(9,1)$ & 7.51 & $\begin{array}{l}4(7 \%) \\
5(93 \%)\end{array}$ & 5 & $\begin{array}{l}8(51 \%) \\
9(49 \%)\end{array}$ & 9 \\
\hline$(9,10)$ & 16.59 & 19 & 19 & 19 & 19 \\
\hline$(10,2)$ & 7.13 & $\begin{array}{l}10(94 \%) \\
11(6 \%)\end{array}$ & 11 & $\begin{array}{l}10(1 \%) \\
11(99 \%)\end{array}$ & 11 \\
\hline$(10,11)$ & 13.36 & 19 & 18 & 19 & 19 \\
\hline$(11,3)$ & 12.97 & $\begin{array}{r}9(18 \%) \\
10(82 \%)\end{array}$ & 10 & $\begin{array}{l}10(34 \%) \\
11(66 \%)\end{array}$ & 11 \\
\hline$(11,12)$ & 5.26 & 16 & 16 & $\begin{array}{l}15(35 \%) \\
16(65 \%)\end{array}$ & 16 \\
\hline$(12,4)$ & 8.89 & $\begin{array}{r}9(35 \%) \\
10(65 \%)\end{array}$ & 10 & $\begin{array}{l}10(78 \%) \\
11(22 \%)\end{array}$ & 10 \\
\hline$(12,13)$ & 7.69 & 11 & 12 & 11 & 12 \\
\hline$(4,5)$ & 2.84 & 7 & 7 & 7 & 8 \\
\hline$(13,6)$ & 10.30 & 5 & 5 & 5 & 5 \\
\hline$(13,7)$ & 14.09 & $\begin{array}{r}9(58 \%) \\
10(42 \%)\end{array}$ & 10 & $\begin{array}{r}9(57 \%) \\
10(43 \%)\end{array}$ & 9 \\
\hline$(7,8)$ & 5.83 & 4 & 3 & 4 & 5 \\
\hline
\end{tabular}

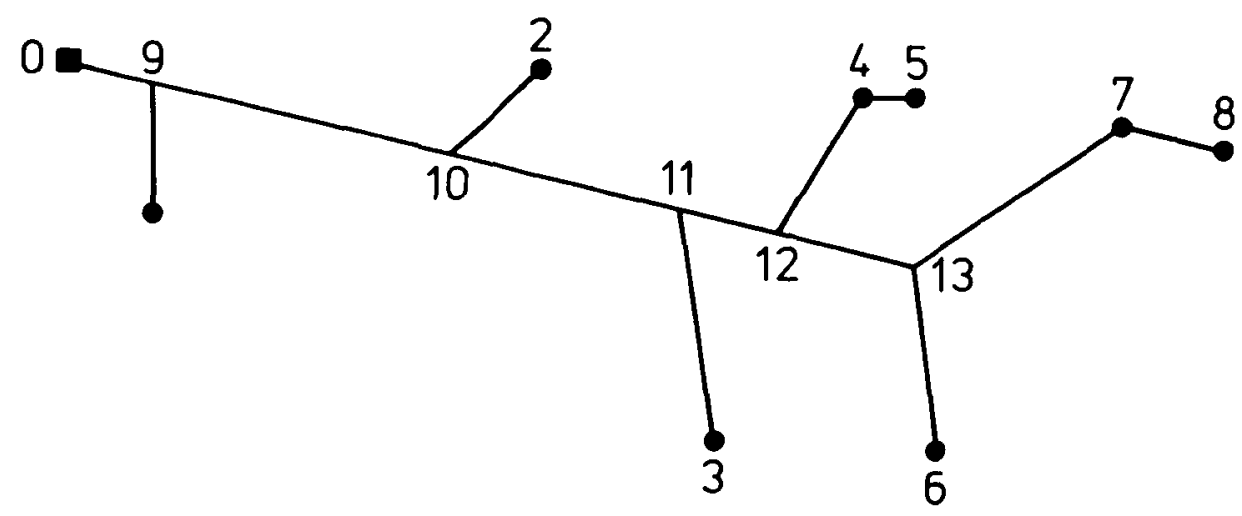

Fig. 3. Tree considered for diameter assignment in example 2. 
system and this involves calculating the various coefficients in the linear programming model. Thus a matrix generator program has to be used to solve LP and IP. The time taken for matrix generation varies between 1 and $2 \mathrm{sec}$ for $T=1$, and between 3 and $10 \mathrm{sec}$ for $T=10$. This time is not included in the execution times given in the tables.

In example 2, the one with the most variables, we only have 133 variables; even much larger problems can be solved, when considering the LP only, which is usually all that is required in practice. Neither storage space required nor computation time appears to be a difficulty in applying this model to practical situations.

\section{Properties of an optimal diameter assignment}

Although the computer program described in the previous sections will produce the optimal set of diameters, it may be useful to gain some insight into the characteristics of an optimal assignment. In this section we therefore state and prove some generally valid properties, which seem to us to be of importance.

We define the average diameter $d$ of a link $(a(j), j)$ by

$$
1 / d^{\alpha_{3}}=\sum_{d \in D}\left(1 / d^{\alpha_{3}}\right) \delta(d, j)
$$

Thus, a pipeline with diameter $d$ would give the same pressure square drop on link $(a(j), j)$ as the combination of pipes with diameter $d$ on a fraction $\delta(d, j)$ (for all $d \in D$ ) of the length of the link.

The cost per unit length, $C(\bar{d})$, corresponding to the average diameter $d$ of link $(a(j), j)$ is given by

$$
C(d)=\sum_{d \in D} C(d) \delta(d, j)
$$

1. In an optimal diameter assignment at most two pipe sizes are used for one link.

Consider the link $(a(j), j)$ and the corresponding optimal diameter selection $\left\{\delta^{*}(d, j)\right\}$. We will show that the set

$$
\left\{d \in D \mid \delta^{*}(d, j) \neq 0\right\}
$$

contains at most two elements.

The average diameter $d$ of the link is given by

$$
1 / \tilde{d}^{\alpha_{3}}=\sum_{d \in D}\left(1 / d^{\alpha_{3}}\right) \delta^{*}(d, j)
$$

Therefore, for link $(a(j), j),\left\{\delta^{*}(d, j)\right\}$ must be a solution of the following linear program in variables $\{\delta(d, j)\}, d \in D$ :

$$
\text { minimize } \sum_{d \in D} C(d) l(j) \delta(d, j)
$$


subject to

$$
\left.\begin{array}{rl}
\sum_{d \in D}\left(1 / d^{\alpha_{3}}\right) \delta(d, j) & =1 / d^{\alpha_{3}}, \\
\sum_{d \in D} \delta(d, j) & =1,
\end{array}\right\}
$$

with

$$
\delta(d, j) \geqslant 0, \quad d \in D
$$

Because the cost row is not proportional to either of the constraint rows, the minimum cannot occur at a non-basic feasible solution of (8). Hence, the minimum occurs only at a basic feasible solution of (8). Thus, an optimal solution has at most two non-zero $\delta(d, j)$ 's.

2. If $C\left(x^{-1 / \alpha_{3}}\right)$ is a discretely strictly convex function of $x$, where

$$
x \in\left\{d_{1}^{-\alpha_{3}}, d_{2}^{-\alpha_{3}}, \ldots, d_{n}^{-\alpha_{3}}\right\},
$$

then the following property holds: If for any link, two pipe sizes are used in the optimal assignment, then they must be of consecutive diameter sizes.

Consider link $(a(j), j)$ and let the corresponding optimal diameter selection have $\delta^{*}\left(d_{1}, j\right) \neq 0, \delta^{*}\left(d_{2}, j\right) \neq 0$. Let $\delta=\delta^{*}\left(d_{1}, j\right)$. Then $1-\delta=\delta^{*}\left(d_{2}, j\right)$. The average diameter $d$ of the link is given by

$$
1 / d^{\alpha_{3}}=\delta / d_{1}^{\alpha_{3}}+(1-\delta) / d_{2}^{\alpha_{3}}
$$

Therefore, for link $(a(j), j)$, the diameters $d_{1}, d_{2}$ must be the solution of the following problem:

minimize $\delta C\left(d_{1}\right)+(1-\delta)\left(C d_{2}\right)$,

$$
\text { where } \delta \text { is given by } \delta / d_{1}^{\alpha_{3}}+(1-\delta) / d_{2}^{\alpha_{3}}=1 / d^{\alpha_{3}}
$$

If $C(d)$ is a discretely strictly convex function of $d^{-\alpha_{3}}$ (or, equivalently, if $C\left(x^{-1 / \alpha_{3}}\right)$ is a discretely, strictly convex function of $x$, where $\left.x \in\left\{d_{1}^{-\alpha_{3}}, \ldots, d_{\alpha}^{-\alpha_{3}}\right\}\right)$ it is obvious from (9) that it is cheapest to choose $d_{1}, d_{2}$ as the consecutive diameters between which $d$ lies.

The condition that $C\left(x^{-1 / \alpha_{3}}\right)$ is a discretely, strictly convex function of $x$ is not a restrictive condition as we now show. This condition is satisfied

(i) if a cost function $C(d)=K d^{\mu}$, where $K, \mu$ are positive constants, is considered, or

(ii) if $C(d)$ is a convex increasing function of $d$.

Finally, we note that for the data we use in Section 4 the condition is also satisfied. 
3. If the specific gravities are the same for all wells then there exists an optimal diameter assignment in which diameters do not decrease in the direction of flow.

The proof of this property, which has been omitted to limit the length of the paper, can be found in [1].

\section{Conclusions}

The algorithm presented here provides an efficient method for finding optimal diameters for a gas pipeline problem and can handle networks of a practical size with little effort. It can therefore be used repeatedly and one can experiment with a large number of trial geometries for the network. The algorithm will give the best diameter and the minimum cost for the geometry considered in a few seconds.

Of course one would like to have a method, other than trial and error, to help in finding the best geometries. An efficient algorithm to find the optimal location of junction points is discussed in [2]; however, the configuration problem is still largely unsolved.

Pipelines are not usually built all at the same time, but over a period of time, as the wells come on stream. The approach used in this paper, to consider the whole pipeline system simultaneously, is therefore a bit unrealistic. Some of the costs are incurred in the future, and one may think of discounting them; it may be cheaper to install two parallel pipes at different times instead of one broader pipe right in the beginning; in general, the whole question of timing the construction of the network has then to be considered. It might also then be economical to install compressors at strategic points in the network to boost the pressure of gas. This gives rise to problems that need quite different models and solution methods to those presented here. But it may well be that the complexity then becomes such that any attempts to apply mathematical optimization techniques will produce few practical, meaningful results.

\section{References}

[1] S. Bhaskaran, Optimal design of gas pipeline networks (Ph.D. Thesis, University of Adelaide, May 1978).

[2] S. Bhaskaran and F. J. M. Salzborn, "Optimal design of gas pipeline networks", submitted for publication to J. Operational Res. Soc.

[3] H. Frank and I. T. Frisch, Communication, transmission and transportation networks (Addison-Wesley, Reading, Mass., 1971).

[4] H. Frank, I. T. Frisch, R. van Slyke and W. S. Chon, "Optimal design of centralized computer networks", Networks 1 (1972), 53-57.

[5] B. Rothfarb, H. Frank, D. M. Rosenbaum, K. Steiglitz and D. J. Kleitman, "Optimal design of offshore national gas pipeline systems", Operations Research 18 (1970), 351-365. 
[6] D. Stephenson, Pipeline design for water engineers (Elsevier, New York, 1976), Chapter 3.

[7] N. Zadeh, "Construction of efficient tree networks: the pipeline problem", Networks 3 (1973), 1-31.

Department of Applied Mathematics

University of Adelaide

Adelaide, S.A. 5001

Australia 